\title{
Potentiality of experimental analysis for characteristics of the Poynting vector components
}

\author{
Khrobatin R., Mokhun I. and Viktorovskaya J. \\ Chernivtsi University, 2 Kotsiubynskiy St., 58012 Chernivtsi, Ukraine \\ e-mail: mokhun@itf.cv.ua
}

Received: 18.03 .2008

After revision: 14.05.2008

\begin{abstract}
Potentiality of experimental analysis for the averaged Poynting vector components is considered. It is shown that joint application of traditional interferometry and Stocks polarimetry should allow for determining unambiguously characteristics of the Poynting vector components at each point of field.
\end{abstract}

Keywords: vortex, polarization singularities, Poynting vector, angular momentum.

PACS: $42.50 . \mathrm{Ct}$

UDC: $535.3,535.4$

One of theoretical aspects in rapidly developing area of the modern optical technology, elaboration of new kinds of optical tweezers [1], is connected with the fact that the vortex beams and polarized waves (both homogeneous and heterogeneous) possess an angular momentum [1-3]. Arising of a controlled angular momentum provides a possibility for controlled rotation of micro-objects locked by corresponding optical traps. Angular momentum of a field may be considered at each spatial point. Averaged angular momentum may also be considered for some spatial areas. It is well-known that the angular momentum may be separated into a spin momentum associated with elliptical polarization, and an orbital one produced by a beam structure (see, e.g., Refs. 2 and 4). However, the density $j_{z}$ of the angular momentum (at least, of its orbital part) is dependent upon the location of the axis $\mathbf{r}=0$ (i.e., the "point of applying"), with regard to which the parameter $j_{z}$ is calculated. As a result, some ambiguity appears. At the same time, another physical value closely associated with the angular momentum, a space distribution of characteristics of the Poynting vector (or, rather, its transverse component), represents a univocal function of coordinates of each field point.

Distribution of parameters of the averaged Poynting vector for Laguerre-Gaussian beams has been considered in [5, 6]. However, the behaviour of Poynting vector has been analyzed only for homogeneously polarized fields and "symmetrical" beams $[2,5]$.

At the same time, the analyzed fields can be more complicated, e.g., when their 
polarization is inhomogeneous. Distribution of transverse component of the Poynting vector for these fields may be characterized by a system of certain points, i.e., a net of Poynting singularities [7]. The importance of these points comes from the fact that the characteristics of this singular net, such as the Poynting field skeleton, determine qualitative behaviour of the Poynting vector at each of the field points [7]. For instance, vortex Poynting singularities [8] are the points, around which the circulation of transverse component of the Poynting vector is observed. In other words, we deal here with the points which are intersections of the plane under analysis and the axis of the angular momentum. Thus, the distribution of characteristics of the Poynting vector components would contain important information on the field, which is concerned with the energy flows.

Nevertheless, one can state that no method for experimental analysis of the Poynting vector components and their singularities has been developed up to now. In the present work we would like to demonstrate that the components of the Poynting vector may be analyzed with traditional optical methods.

It has been shown $[3,7]$ that the instantaneous components of the Poynting vector may be written as

$$
\left\{\begin{array}{c}
P_{x} \approx-\frac{c}{4 \pi k}\left\{E_{x} T_{2}-E_{y} T_{1}\right\} \\
P_{y} \approx-\frac{c}{4 \pi k}\left\{E_{y} T_{2}+E_{x} T_{1}\right\}, \\
P_{z} \approx \frac{c}{4 \pi}\left\{E_{x}^{2}+E_{y}^{2}\right\}
\end{array}\right.
$$

where

$$
\begin{aligned}
& \left\{\begin{array}{l}
T_{1}=E_{x} \Phi_{x}^{y}-E_{y} \Phi_{y}^{x}+\frac{A_{x}^{y}}{A_{x}} E_{x, \frac{\pi}{2}}-\frac{A_{y}^{x}}{A_{y}} E_{y, \frac{\pi}{2}}, \\
T_{2}=E_{x} \Phi_{x}^{x}+E_{y} \Phi_{y}^{y}+\frac{A_{x}^{x}}{A_{x}} E_{x, \frac{\pi}{2}}+\frac{A_{y}^{y}}{A_{y}} E_{y, \frac{\pi}{2}}
\end{array}\right. \\
& \left\{\begin{array}{l}
E_{i}=A_{i} \cos \left(\omega t+\Phi_{i}-k z\right) \\
E_{i, \frac{\pi}{2}}=A_{i} \sin \left(\omega t+\Phi_{i}-k z\right),
\end{array}\right.
\end{aligned}
$$

$A_{i}$ and $\Phi_{i}(i=x, y)$ denote respectively the amplitudes and phases of the corresponding field components, $A_{i}^{l}$ and $\Phi_{i}^{l}$ are their partial derivatives, $i, l=x, y$ and $k=\omega / c$ represents the wave number. Note that here the axis $z$ coincides with a preferential direction of the wave propagation.

One can show after some algebraic transformations that the averaged components of the Poynting vector are as follows: 


$$
\left\{\begin{array}{c}
\left.\bar{P}_{x} \approx-\frac{c}{8 \pi k}\left\{\left[A_{x}^{2} \Phi_{x}^{x}+A_{y}^{2} \Phi_{y}^{x}\right]-A_{x} A_{y}\left(\Phi_{x}^{y}-\Phi_{y}^{y}\right) \cos \Delta-\left(A_{x} A_{y}^{y}+A_{y} A_{x}^{y}\right) \sin \Delta\right]\right\} \\
\left.\bar{P}_{y} \approx-\frac{c}{8 \pi k}\left\{\left[A_{x}^{2} \Phi_{x}^{y}+A_{y}^{2} \Phi_{y}^{y}\right]+A_{x} A_{y}\left(\Phi_{x}^{x}-\Phi_{y}^{x}\right) \cos \Delta+\left(A_{x} A_{y}^{x}+A_{y} A_{x}^{x}\right) \sin \Delta\right]\right\} . \\
\bar{P}_{z} \approx \frac{c}{8 \pi}\left\{A_{x}^{2}+A_{y}^{2}\right\}
\end{array}\right.
$$

The second and third terms in the braces appearing in the r. h. s. of the first two rows of Eq's. (4) may be rewritten as follows:

$$
A_{x} A_{y}\left(\Phi_{x}^{i}-\Phi_{y}^{i}\right) \cos \Delta+\left(A_{x} A_{y}^{i}+A_{y} A_{x}^{i}\right) \sin \Delta=\frac{\partial}{\partial i}\left(A_{x} A_{y} \sin \Delta\right),
$$

where $i=x, y$. Then the system of Eqs. (4) is transformed to

$$
\left\{\begin{array}{c}
\bar{P}_{x} \approx-\frac{c}{8 \pi k}\left\{\left[A_{x}^{2} \Phi_{x}^{x}+A_{y}^{2} \Phi_{y}^{x}\right]-\frac{\partial}{\partial y}\left(A_{x} A_{y} \sin \Delta\right)\right\} \\
\bar{P}_{y} \approx-\frac{c}{8 \pi k}\left\{\left[A_{x}^{2} \Phi_{x}^{y}+A_{y}^{2} \Phi_{y}^{y}\right]+\frac{\partial}{\partial x}\left(A_{x} A_{y} \sin \Delta\right)\right\} . \\
\bar{P}_{z} \approx \frac{c}{8 \pi}\left\{A_{x}^{2}+A_{y}^{2}\right\}
\end{array}\right.
$$

It follows from Eqs. (6) that one of the possible ways for measuring the components of the Poynting vector is a complete analysis of the orthogonal components, namely:

(i) measurements of intensities of the components (in order to determine the component amplitudes and their derivatives);

(ii) phasometry (interferometry) of the components (in order to determine the component phases and their derivatives.

However, the phase difference $\Delta$ between the orthogonal components is also included in Eqs. (6). Let us stress the following points:

(i) as known from the work [9], measurements of the "absolute" phase in optics is "problematic". Only the phase difference between the object wave and the reference one is fixed. In other words, the phase is measured up to a constant additive term.

(ii) it is practically impossible to create experimental setup for measuring simultaneously the phases of the components with the same constant additives.

Consequently, the phase difference $\Delta$ cannot be measured with a sufficient accuracy using the analysis of the components.

However, this parameter could be easily measured by means of Stokes polarimetry [10]. The Stokes parameters for any monochromatic wave acquire the form (see [9])

$$
s_{0}=A_{x}^{2}+A_{y}^{2}, \quad s_{1}=A_{x}^{2}-A_{y}^{2}, \quad s_{2}=2 A_{x} A_{y} \cos \Delta, \quad s_{3}=2 A_{x} A_{y} \sin \Delta .
$$

The $\Delta$ value may be determined as

$$
\Delta=\arctan \frac{s_{3}}{s_{2}} \text {. }
$$


Then Eqs. (6) may be used when analyzing the Poynting vector components. Taking Eqs. (7) into account, we transform Eqs. (6) to finally obtain

$$
\left\{\begin{array}{c}
\bar{P}_{x} \approx-\frac{c}{16 \pi k}\left\{\left[\left(s_{0}+s_{1}\right) \Phi_{x}^{x}+\left(s_{0}-s_{1}\right) \Phi_{y}^{x}\right]-\frac{\partial s_{2}}{\partial y}\right\} \\
\bar{P}_{y} \approx-\frac{c}{16 \pi k}\left\{\left[\left(s_{0}+s_{1}\right) \Phi_{x}^{y}+\left(s_{0}-s_{1}\right) \Phi_{y}^{y}\right]+\frac{\partial s_{2}}{\partial x}\right\} \\
\bar{P}_{z} \approx \frac{c}{8 \pi} s_{0}
\end{array}\right.
$$

Thus, the Poynting vector components are defined by the Stocks parameters and the derivatives of the component phases. Note that only one component (to be specific, $y$ component) is required for the phasometry, because the phase of the other component is defined as $\Phi_{x}=\Delta+\Phi_{y}$. Obviously, the phasometry of 'smooth' waves (i.e., those including no singularities in the area under analysis) is preferable in any practical situation.

It is known (see, e.g., Ref. 11, whose subject is closest to that of our work) that the three types of measurements are necessary in order to determine the phase of scalar field at each point of that field, which result in the following: (i) intensity of the component field, $I_{i}=A_{i}^{2}$, (ii) intensity of the reference wave, $I_{r}=A_{r}^{2}$, and (iii) intensity of the total field, $I_{s}$. The component phase (up to the constant additive) may be derived from the relation

$$
\Phi_{i}=\arccos \frac{I_{s}-\left(I_{i}+I_{r}\right)}{2 \sqrt{I_{i} I_{s}}} .
$$

Naturally, the phase derivatives $\Phi_{i}^{k}$ included in Eqs. (6) and (9) are independent of the constant additives.

It is worth mentioning that the accuracy of the phase measurements for the components may be essentially increased if one uses a two-beam interferometric technique, in which a reference wave with the phase shifted by $\chi$ is mixed with the component field [11]. In this case five measurements are made, providing the intensities of the component alone and the reference wave alone, as well as the mixed waves with $\chi=0$ and $\chi= \pm \pi / 2$. This leads to a substantial degree of redundancy, which is helpful for reducing experimental errors.

In conclusion, we are in a position to state that joint application of the traditional interferometry and the Stokes polarimetry should enable one to determine unambiguously the characteristics of the Poynting vector components at each field point.

\section{References}

1. Lang M J AND Block S M, 2003. Resource Letter: LBOT-1: Laser-based optical tweezers. Am. J. Phys. 71: 201-215. 
2. Allen L, Padgett M J and Babiker M. The orbital angular momentum of light. E Wolf Progress in Optics XXXIX Elsevier Science B.V. (1999).

3. Mokhun I, Brandel $\mathrm{R}$ and Viktorovskaya Ju, 2006. Angular momentum of electromagnetic field in areas of polarization singularities. Ukr. J. Phys. Opt. 7: 6373.

4. Berry M V, 1998. Paraxial beams of spinning light. Proc. SPIE. 3487: 6-11.

5. Allen L, Padgett M J, 2000. The Poynting vector in Laguerre-Gaussian beams and the interpretation of their angular momentum density. Opt. Comm. 184: 67-71.

6. Berry M V, 2005. Phase vortex spirals. J. Phys. A. 38: L745-L751.

7. Mokhun I. Introduction to linear singular optics. Chapter 1. in Optical correlation techniques and applications ed. by O.V.Angelsky SPIE press Bellingham Washington USA: 1-132. (2007).

8. Mokhun I, Khrobatin R, Mokhun A and Viktorovskaya Ju, 2007. The behavior of the Poynting vector in the area of elementary polarization singularities. Opt. Applicata. XXXVII: 261-277.

9. Born $M$ and Wolf E. Principles of Optics. $6^{\text {th }}$ (corrected) edition. Pergamon Press, Oxford. (1987).

10. Azzam R M A and Bashara N M. Ellipsometry and Polarized Light. North-Holand (1977).

11. Shvartsman N and Freund I, 1995. Speckle spots ride phase saddles side saddle. Opt. Comm. 117: 228-234. 